\title{
Bayer Patterned Image Compression Based on Structure Conversion and APBT
}

\author{
Chengyou Wang*, Songzhao Xie and Xiao Zhou \\ School of Mechanical, Electrical and Information Engineering, Shandong University, \\ Weihai 264209, China \\ wangchengyou@sdu.edu.cn,xiesongzhao@hotmail.com,zhouxiao@sdu.edu.cn
}

\begin{abstract}
The color filter array (CFA) captures only one-third of the necessary color intensities and the full color image is generated from the captured data by interpolation. In recent years, the algorithm of Bayer patterned image compression based on "structure conversion" has achieved better image quality. On the basis of previous work, a new algorithm based on the all phase biorthogonal transform (APBT) and all phase IDCT (APIDCT) interpolation is proposed in this paper. Instead of the conventional JPEG compression, APBT is applied to the JPEG image compression (APBT-JPEG), which significantly reduces complex multiplications and makes the quantization table easier. In the interpolation step, APIDCT interpolation method is introduced. Experimental results show that the proposed algorithm outperforms the one based on "structure conversion"; and the APIDCT interpolation performs close to the conventional interpolation methods. Therefore, the algorithm proposed in this paper is more suitable for Bayer image compression.
\end{abstract}

Keywords: Image Compression, All Phase Biorthogonal Transform (APBT), Structure Conversion, Color Filter Array (CFA)

\section{Introduction}

From a color filter array (CFA), digital cameras obtain the color image information. However, only one color component is retained at each pixel location. To recover the full color image, interpolation is applied to the decompressed image. In various structures of CFA, Bayer pattern is the most widely used, as shown in Figure 1 [1]. It can be seen that the number of green $(\mathrm{G})$ component is twice than the red $(\mathrm{R})$ or blue $(\mathrm{B})$ component, which is more suitable for the color sensitivity of human eyes.

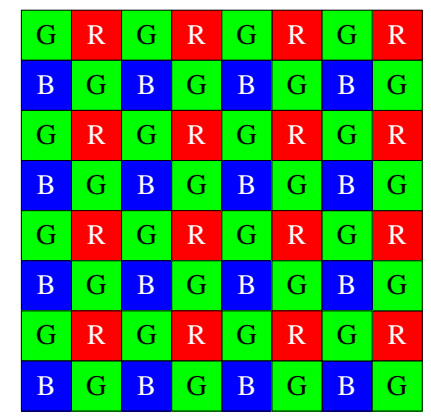

Figure 1. Bayer patterned CFA structure 
Since each pixel and its adjacent pixels in space are not continuous for Bayer image, the high frequency component is large between the adjacent components. Direct compression to the Bayer image does not make for improving the compression ration. Therefore, various compression algorithms for Bayer image have appeared. Lee [2] proposed "geometric rotation" compression method, and the image data is firstly converted from RGB color space to $\mathrm{YCbCr}$ color space. The $\mathrm{Cb}, \mathrm{Cr}$ components are equivalent to a 4:2:0 sampling operation, while $\mathrm{Y}$ component needs to be converted into rhombus with a $45^{\circ}$ rotation. In recent years, Koh [3] proposed two new lossy compression algorithms, referred to as "structure conversion" and "structure separation", which have achieved better results. On this basis, Xie [4] improved the structure conversion algorithm. In addition, there are lots of algorithms for original data compression [5-7]. However, all these algorithms used discrete cosine transform (DCT) in compression step, which brought more complex quantization table and serious blocking artifacts at low bit rates.

To solve these problems, Ref. [8] proposed the new concept of the all phase biorthogonal transform (APBT), which had been successfully applied to JPEG image compression (APBT-JPEG). On the basis of APBT theory, a novel Bayer patterned image compression algorithm based on APBT-JPEG and all phase IDCT (APIDCT) interpolation is proposed in this paper. In the compression step, APBT is adopted instead of the conventional DCT, and the uniform quantization table is used instead of the complex quantization Table, which makes the computation complexity reduced and the hardware easier implemented. Besides, a novel interpolation method of APIDCT [9] is used to the depressed image data. Experimental results show the algorithm proposed in this paper performs better than the conventional algorithms.

The rest of this paper is organized as follows. Section 2 introduces the structure conversion algorithm for Bayer patterned image. In Section 3, the Bayer image compression algorithm based on structure conversion and APBT is presented. In Section 4, experimental results and comparisons with conventional algorithms are presented. Finally, conclusions and remarks on further research are given in Section 5.

\section{Structure Conversion Algorithm for Bayer Patterned Image}

The scheme of the improved structure conversion algorithm in [3] is shown in Figure 2. First, the structure conversion is applied to $\mathrm{G}$ component, and then data in RGB color space are converted to $\mathrm{YCbCr}$ color space, because the correlation between pixels in $\mathrm{RGB}$ color space is higher than that in $\mathrm{YCbCr}$ color space. Due to the original rectangular distribution of $\mathrm{R}$ and $\mathrm{B}$ components, the loose rectangle only needs to become compact rectangle without low-pass filtering and structure conversion.

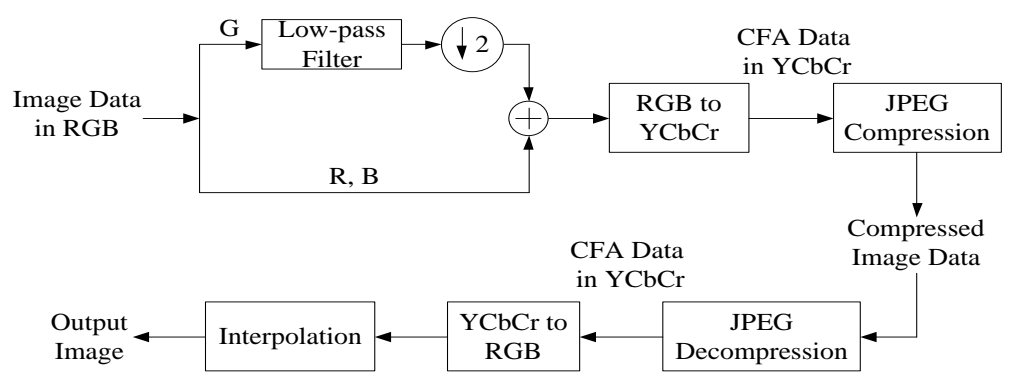

Figure 2. Scheme of Structure Conversion Algorithm 


\subsection{Color Space Conversion}

The original Bayer image can be seen as a set composed of GB/RG blocks with size of $2 \times 2$. Using the similarity between pixels, R, G and B components can be converted to luminance and chrominance values in $\mathrm{YCbCr}$ color space by transform matrix, as shown in Figure 3. The $\mathrm{Cb}$ and $\mathrm{Cr}$ components are equivalent to a 4:2:0 sampling operation. The color space transform matrix is:

$$
\left[\begin{array}{c}
\mathrm{Y}^{\mathrm{ul}} \\
\mathrm{Y}^{\mathrm{lr}} \\
\mathrm{Cb} \\
\mathrm{Cr}
\end{array}\right]=\left[\begin{array}{cccc}
128.6 & 0 & 25 & 65.5 \\
0 & 128.6 & 25 & 65.5 \\
-37.1 & -37.1 & 112 & -37.8 \\
-46.9 & -46.9 & -18.2 & 112
\end{array}\right]\left[\begin{array}{c}
\mathrm{G}^{\mathrm{ul}} \\
\mathrm{G}^{\mathrm{lr}} \\
\mathrm{B} \\
\mathrm{R}
\end{array}\right]+\left[\begin{array}{c}
0 \\
0 \\
128 \\
128
\end{array}\right]
$$

where ${ }^{\text {ul }}$ represents upper left corner, while ${ }^{\text {lr }}$ represents lower right corner.

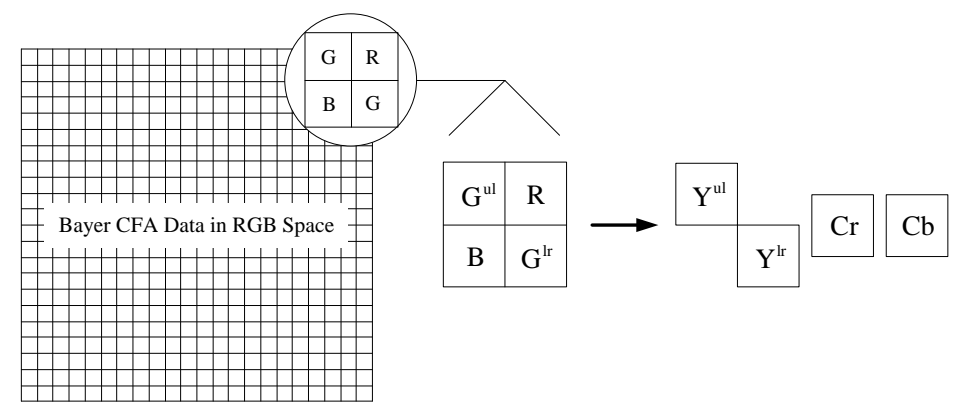

Figure 3. Blocks of Color Space Conversion

\subsection{Structure Conversion}

The structure conversion of G component is shown in Figure 4, where Go, Ge and Gc are odd, even, and converted columns respectively. Since the G component is quincuncial, it needs to become a rectangle. Directly emerging odd and even columns will produce a lot of pseudo-high-frequency components, in order to suppress the generation of pseudo-high-frequency components, G component needs a smoothing processing, which can be divided into two steps: low-pass filter and 2:1 sampling of columns. The impulse response function of the low-pass filter is:

$$
h_{d}[m, n]=\frac{1}{4}\left[\begin{array}{lll}
0 & 0 & 1 \\
0 & 2 & 4 \\
0 & 0 & 1
\end{array}\right]
$$

After the sampling of columns, $G$ component becomes a rectangle, of which the number of rows is equal to the original image and the number of columns becomes a half of the original number of columns.

The image data achieved after structure conversion and color space conversion is compressed by the still image compression standard JPEG. After the storage or transmission, the compressed image is decompressed to recover the CFA data, of which the steps are opposite to ones described earlier. After decompression, there is no need to add the inverse transform of the filtering, because the correlation between pixels has been weak after a previous process. This not only makes the decompression algorithm easier, but also achieves better results through the practical test. 


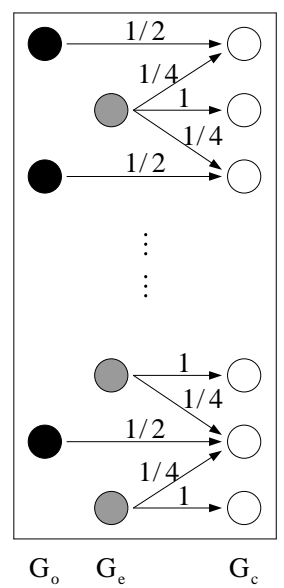

(a)

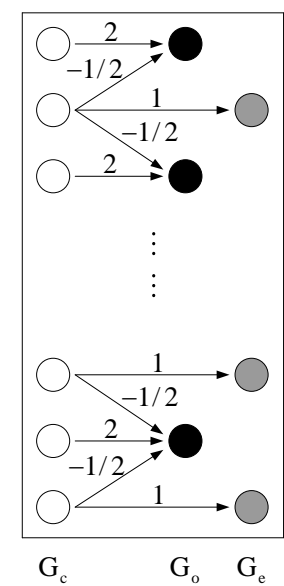

(b)

Figure 4. Structure Conversion

\section{Bayer Patterned Image Compression Based on Structure Conversion and APBT}

The process of the new algorithm proposed in this paper is shown in Figure 5. Wherein, the steps of structure conversion and color space conversion are same as ones of the structure conversion algorithm for original Bayer image, while the second half of the algorithm is image compression based on APBT instead of the JPEG compression in the conventional algorithm. Meanwhile, APIDCT interpolation is applied to the reconstructed CFA data to recover the full color image.

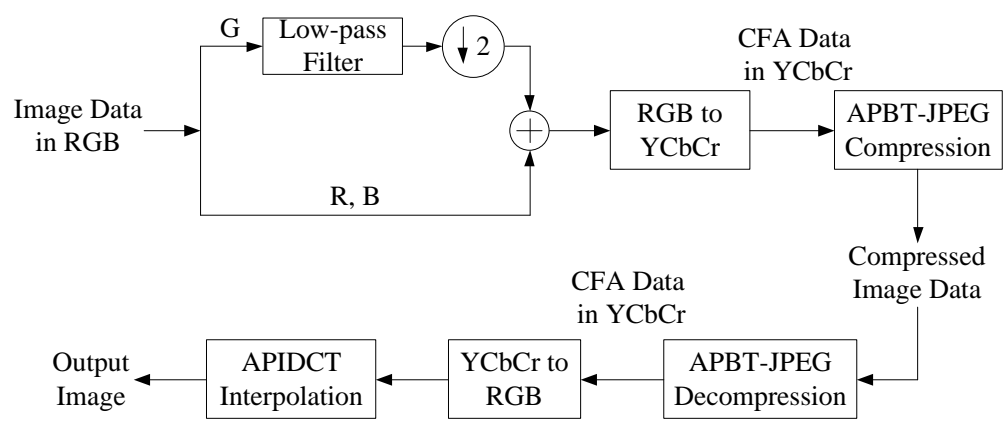

\section{Figure 5. Scheme of Bayer Patterned Image Compression based on Structure Conversion and APBT}

\subsection{APBT-JPEG Compression Algorithm}

Figure 6 shows the APBT-JPEG compression algorithm. Substantially the same as basic steps of the DCT-based JPEG image compression algorithm, but the APBT replaces the conventional DCT and the simple uniform quantization step is applied instead of the complex quantization table. Based on different orthogonal transforms: Walsh-Hadamard, DCT and IDCT, three kinds of all phase biorthogonal transform (APBT) were presented, referred to as all phase Walsh biorthogonal transform (APWBT), all phase discrete cosine biorthogonal transform (APDCBT) and all phase inverse discrete cosine biorthogonal transform (APIDCBT). 


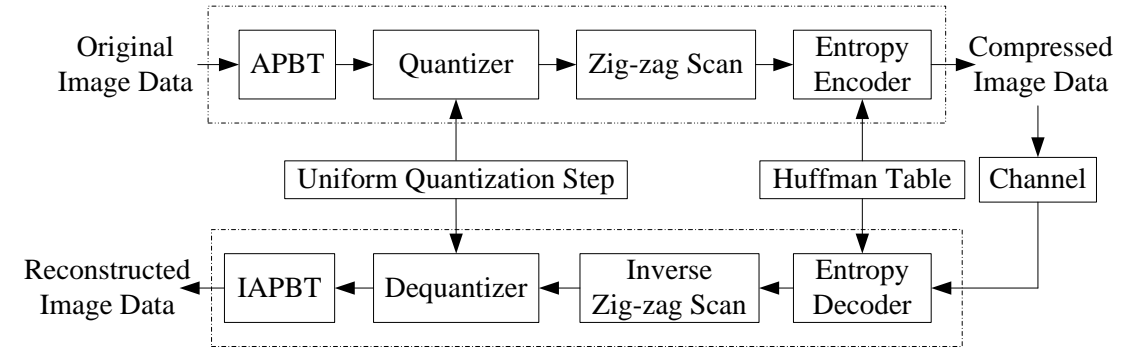

Figure 6. Diagram of the APBT-JPEG Compression Algorithm

\subsection{Design of All Phase IDCT Interpolation}

Based on the all phase filtering theory [10], the APIDCT filter is proposed in [9]. Let $\boldsymbol{h}_{(2 N-1) \times(2 N-1)}$ denote the 2-D unit impulse response of the all phase sequency filtering, and $\boldsymbol{F}_{N \times N}$ denote the sequency response vector of length $N$. Therefore, the design of 2-D APIDCT filter with size of $(2 N-1) \times(2 N-1)$ is composed of (3) and (4)

$$
\begin{gathered}
{\left[\boldsymbol{h}_{1 / 4}\right]_{N \times N}=\boldsymbol{V F}_{N \times N} \boldsymbol{V}^{\mathrm{T}},} \\
h(m, n)=h(-m, n)=h(m,-n)=h(-m,-n), m, n=0,1, \cdots, N-1,
\end{gathered}
$$

where $\boldsymbol{V}$ denotes the APIDCBT matrix [8], and has the form

$$
V(m, n)=\left\{\begin{array}{lc}
\frac{1}{N}, & m=0, n=0,1, \cdots, N-1, \\
\frac{N-m+\sqrt{2}-1}{N^{2}} \cos \frac{m(2 n+1) \pi}{2 N}, & m=1,2, \cdots, N-1, n=0,1, \cdots, N-1 .
\end{array}\right.
$$

When $N=4$ and $N=2$, the APIDCT filters used in this paper are

$$
\boldsymbol{h}_{7 \times 7}=\left[\begin{array}{ccccccc}
0 & 0.01 & 0 & 0.01 & 0 & 0.01 & 0 \\
0.01 & 0 & -0.06 & 0 & -0.06 & 0 & 0.01 \\
0 & -0.06 & 0 & 0.34 & 0 & -0.06 & 0 \\
0.01 & 0 & 0.34 & 1 & 0.34 & 0 & 0.01 \\
0 & -0.06 & 0 & 0.34 & 0 & -0.06 & 0 \\
0.01 & 0 & -0.06 & 0 & -0.06 & 0 & 0.01 \\
0 & 0.01 & 0 & 0.01 & 0 & 0.01 & 0
\end{array}\right] \text {, }
$$

and

$$
\boldsymbol{h}_{3 \times 3}=\left[\begin{array}{ccc}
0 & 0.25 & 0 \\
0.25 & 1 & 0.25 \\
0 & 0.25 & 0
\end{array}\right] .
$$

From (6) and (7), it can be seen that the sum of weight coefficients (except the center one) is 1 in each interpolation template and the value of the central symmetric weight coefficients is 0 alternately. In this paper, $\boldsymbol{h}_{7 \times 7}$ is suitable for $\mathrm{G}$ component. When interpolating the R or B component, the APIDCT filters are $\boldsymbol{h}_{11 \times 11}^{\prime}$ and $\boldsymbol{h}_{3 \times 3}^{\prime}$, which can 
be obtained by $\boldsymbol{h}_{7 \times 7}$ and $\boldsymbol{h}_{3 \times 3}$ rotating $45^{\circ}$ respectively. Taking into account the characteristics of the data, the $\mathrm{R}$ or $\mathrm{B}$ component is firstly interpolated by the filter $\boldsymbol{h}_{11 \times 11}^{\prime}$, and then the filter $\boldsymbol{h}_{3 \times 3}^{\prime}$ is applied to the obtained data.

\section{Experimental Results}

This paper selects the image Lena with size of $512 \times 512$ for test, and the Bayer CFA data needed in the algorithm can be obtained by down-sampling the 24bits/pixel full color images. In the structure conversion algorithm, bilinear interpolation is adopted.

\subsection{Comparison of CPSNR}

To measure the performance of algorithm proposed in this paper, we choose the Composite Peak Signal to Noise Ratio (CPSNR) [11] defined as

$$
\mathrm{CPSNR}=10 \log _{10}\left[\frac{255^{2}}{\frac{1}{3 M N} \sum_{k=1}^{3} \sum_{i=1}^{M} \sum_{j=1}^{N}\left[I_{\mathrm{in}}(i, j, k)-I_{\text {out }}(i, j, k)\right]^{2}}\right](\mathrm{dB}),
$$

where $\boldsymbol{I}_{\text {in }}$ and $\boldsymbol{I}_{\text {out }}$ are the original and reconstructed images respectively, $M$ and $N$ are the dimensions of each color component array, $i$ and $j$ are the locations of pixels in the color plane, and $k$ represents the color plane.

The comparison results of the objective quality of the two algorithms are shown in Table 1. From the data in the table, it can be seen that the proposed algorithm in this paper is better than the conventional structure conversion algorithm at various bit rates (0.2bpp 1.0bpp), in terms of CPSNR. Especially, for the Bayer images dominated by low-frequency components, the CPSNRs are improved more obviously.

Table 1. Comparison of CPSNR at Various Bit Rates Applied to Lena

\begin{tabular}{|c|c|c|}
\hline \multirow{2}{*}{ Bit rate/bpp } & \multicolumn{2}{|c|}{ CPSNR/dB } \\
\cline { 2 - 3 } & Structure conversion & Proposed algorithm \\
\hline 0.20 & 27.04 & 27.55 \\
\hline 0.30 & 29.22 & 29.41 \\
\hline 0.40 & 30.21 & 30.39 \\
\hline 0.50 & 30.83 & 31.01 \\
\hline 0.60 & 31.23 & 31.43 \\
\hline 0.70 & 31.54 & 31.75 \\
\hline 0.80 & 31.81 & 32.02 \\
\hline 1.00 & 32.14 & 32.39 \\
\hline
\end{tabular}

\subsection{Comparison of Subjective Quality}

Figure 7 gives the reconstructed images of Lena at $0.20 \mathrm{bpp}$ by using the structure conversion algorithm and the proposed algorithm in this paper. From the reconstructed images, we can see that the visual effect of the proposed algorithm is better than the conventional structure conversion algorithm. Further, the edge information of the image is clearer and easier to be accepted without blocking artifacts. 


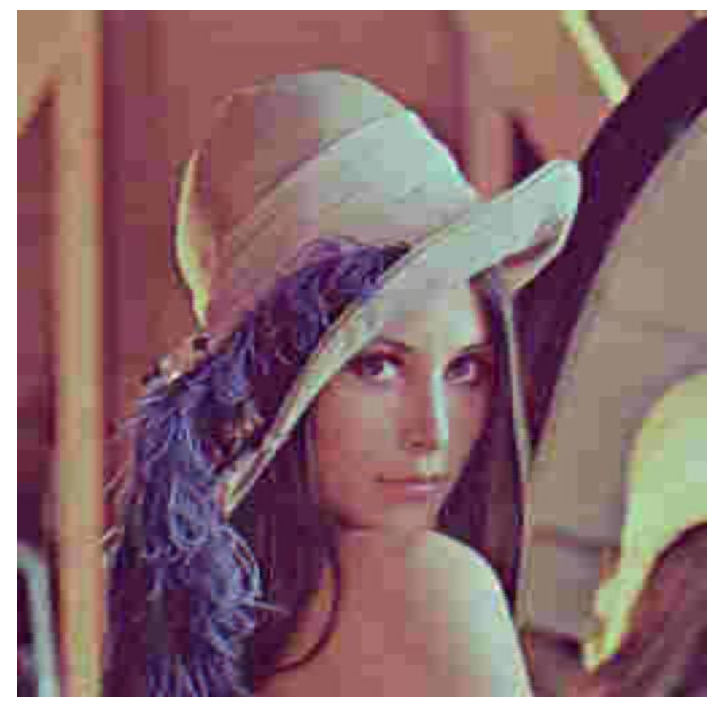

(a)

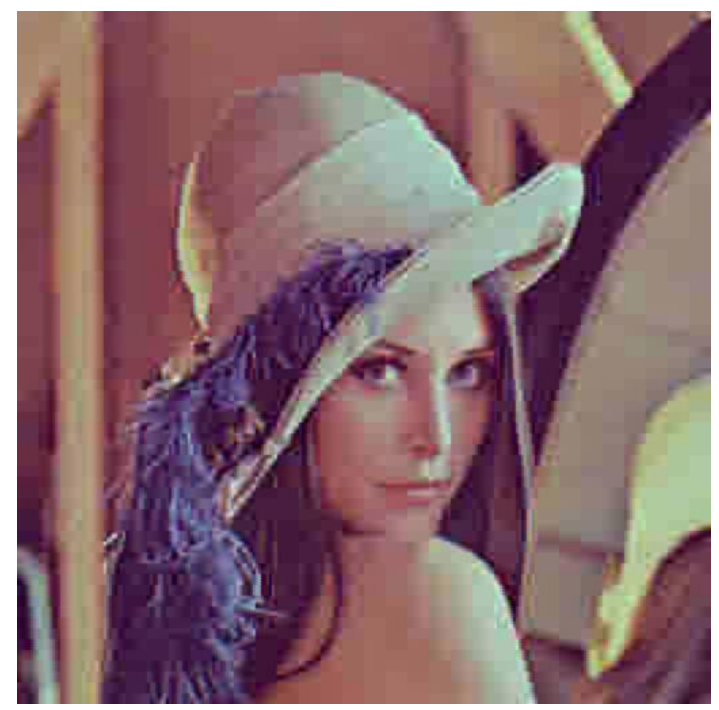

(b)

Figure 7. The Reconstructed Images of Lena (0.20bpp): (a) Improved Structure Conversion, (b) Proposed Algorithm

\section{Conclusion}

This paper presents a novel algorithm for Bayer image compression based on the structure conversion and APBT. From the simulation experiments, it can be concluded that the CPSNR of the proposed algorithm in this paper outperforms the conventional structure conversion algorithm, and better visual quality also has been achieved. Thus we can foresee that the proposed algorithm can be widely used in Bayer image compression.

\section{Acknowledgements}

This work was supported by the promotive research fund for excellent young and middle-aged scientists of Shandong Province, China (Grant No. BS2013DX022) and the National Natural Science Foundation of China (Grant No. 61201371). The authors would like to thank Xiaoyan Wang, Fanfan Yang and Qiming Fu for their kind help and valuable suggestions. The authors also thank the anonymous reviewers and the editors for their valuable comments to improve the presentation of the paper.

\section{References}

[1] R. Lukac, K. N. Plataniotis, and D. Hatzinakos, "Color image zooming on the Bayer pattern", IEEE Transactions on Circuits and Systems for Video Technology, vol. 15, no. 11, (2005), pp. 1475-1491.

[2] S. Y. Lee and A. Ortega, "A novel approach of image compression in digital cameras with a Bayer color filter array", Proceedings of the IEEE International Conference on Image Processing, Thessaloniki, Greece, vol. 3, (2001) October 7-10, pp. 482-485.

[3] C. C. Koh, J. Mukherjee, and S. K. Mitra, "New efficient methods of image compression in digital cameras with color filter array", IEEE Transactions on Consumer Electronics, vol. 49, no. 4, (2003), pp. 1448-1456.

[4] X. Xie, G. L. Li, and Z. H. Wang, "A low complexity and high efficient method for image compression with Bayer CFAs", Tsinghua Science and Technology, vol. 12, no. 1, (2007), pp. 22-29.

[5] N. Zhang and X. L. Wu, "Lossless compression of color mosaic images", IEEE Transactions on Image Processing, vol. 15, no. 6, (2006), pp. 1379-1388. 
[6] K. H. Chung and Y. H. Chan, "A lossless compression scheme for Bayer color filter array images", IEEE Transactions on Image Processing, vol. 17, no. 2, (2008), pp. 134-144.

[7] A. Bazhyna and K. Egiazarian, "Lossless and near lossless compression of real color filter array data", IEEE Transactions on Consumer Electronics, vol. 54, no. 4, (2008), pp. 1492-1500.

[8] Z. X. Hou, C. Y. Wang, and A. P. Yang, "All phase biorthogonal transform and its application in JPEG-like image compression”, Signal Processing, Image Communication, vol. 24, no. 10, (2009), pp. 791-802.

[9] Z. X. Hou, X. J. Guo, and X. Yang, "A novel hierarchical coding algorithm based on multi-subsample and the all phase IDCT interpolation", Proceedings of the SPIE Mathematics of Data/Image Coding, Compression, and Encryption VII, with Applications, Denver, CO, USA, vol. 5561, (2004) August 4-5, pp. 76-83.

[10]Z. X. Hou and X. Yang, "The all phase DFT filter", Proceedings of the 10th IEEE Digital Signal Processing Workshop and the 2nd IEEE Signal Processing Education Workshop, Pine Mountain, Georgia, USA, (2002) October 13-16, pp. 221-226.

[11] J. Mukherjee, M. K. Lang and S. K. Mitra, "Demosaicing of images obtained from single-chip imaging sensors in YUV color space”, Pattern Recognition Letters, vol. 26, no. 7, (2005), pp. 985-997.

\section{Authors}

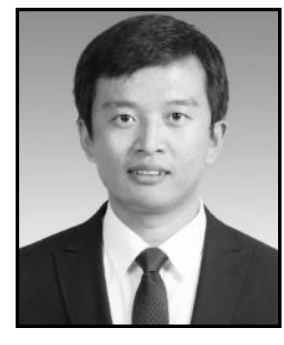

Chengyou Wang was born in Shandong province, China in 1979. He received his B.E. degree in electronic information science and technology from Yantai University, China, in 2004 and his M.E. and Ph.D. degree in signal and information processing from Tianjin University, China, in 2007 and 2010 respectively. Now he is an associate professor in the School of Mechanical, Electrical and Information Engineering, Shandong University, Weihai, China. His current research interests include image processing and transmission techniques, multidimensional signal and information processing, and smart grid technology.

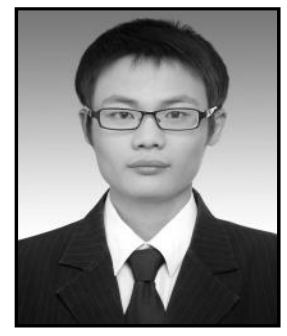

Songzhao Xie was born in Shandong province, China in 1988. He received his B.S. degree in electronic information science and technology from Shandong University, Weihai, China, in 2011, and his M.E. degree in circuits and systems from Shandong University, China, in 2014. Now he is with the Huawei Technologies Co. Ltd., Nanjing, China. His research interests concentrate on image compression.

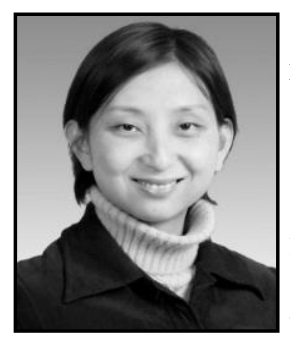

Xiao Zhou was born in Shandong province, China in 1982. She received her B.E. degree in automation from Nanjing University of Posts and Telecommunications, China, in 2003, her M.E. degree in information and communication engineering from Inha University, Korea in 2005, and her Ph.D. degree in information and communication engineering from Tsinghua University, China in 2013. Now she is a lecturer in the School of Mechanical, Electrical and Information Engineering, Shandong University, Weihai, China. Her current research interests include wireless communication technology, image processing and transmission technology. 